\title{
The effects of aging on selectivity and control in short-term recall
}

\author{
ALAN D. CASTEL \\ University of Toronto, Toronto, Ontario, Canada \\ AARON S. BENJAMIN \\ University of Illinois at Urbana-Champaign, Champaign, Illinois \\ FERGUS I. M. CRAIK \\ Rotman Research Institute at Baycrest Centre for Geriatric Care, Toronto, Ontario, Canada \\ and \\ MICHAEL J. WATKINS \\ Rice University, Houston, Texas
}

\begin{abstract}
The ability to control encoding and retrievalprocesses strategicallyis criticalfor the efficient use of memory. We examined the ability of younger and older adults to selectively remember words on the basis of their arbitrary point values by using a technique developed by Watkins and Bloom (1999). In the first three experiments, younger subjects recalled more words than did older subjects, but an independent index of recall selectivity showed that older subjects were apparently more successful in selecting higher valued words. However, a fourth experiment showed that this superior selectivity on the part of older adults was attributable to their greater proportional reliance on primary memory recall. Overall, the data suggest that although older adults recall fewer words than do younger adults, they exert as much control over some aspects of encoding.
\end{abstract}

The ability to control what information from our immediate environment we will retain for future use is a critical aspect of the efficient use of human memory. Although it is well known that aging impairs the ability to encode and retrieve certain types of information (see Kester, Benjamin, Castel, \& Craik, 2002, for a recent review), the degree to which aging affects the strategic control of memory is less well understood. The conscious control of memory processes determines the selection of information to be remembered, how that information is perceived, and how well the information is accessed later in time.

The effects of aging on the ability to control memory processes have been examined in several lines of research. Zacks, Radvansky, and Hasher (1996) used a directed forgetting paradigm, in which younger and older adults were presented with words, each of which was followed by a cue to remember or to forget that word. At the end of the

This research was supported by a grant from the Natural Sciences and Engineering Research Council of Canada to F.I.M.C. Portions of this research were presented at the 42nd Annual Meeting of the Psychonomic Society, November 2001. Parts of this research were carried out while A.S.B. was at the Rotman Research Institute. We thank Larry Jacoby and two anonymous reviewers for their helpful comments and suggestions regarding this manuscript. We also thank Sharyn Kreuger, Jane Logan, and Nicole Jordan for their assistance with the experiments. Correspondence should be addressed to A. Castel, Department of Psychology, University of Toronto, Toronto, ON, M5S 3G3 Canada (e-mail: alan@psych.utoronto.ca). list, subjects were asked to recall only the items that they were instructed to remember. Compared with younger adults, older adults recalled fewer words that they had been instructed to remember but more words that they had been instructed to forget. This finding suggests that older adults have less control over memory.

Another way in which impairments of cognitive control affect older adults' memory performance is by reducing the degree to which their memory is guided by conscious recollection. Researchers who have attempted to disentangle conscious recollection from automatic processes have shown that older adults tend to rely on automatic, or familiarity-based memory processing and that conscious recollection is reduced with age (Jacoby \& Hay, 1998). This observation is consistent with results from the remember/know paradigm, in which subjects are asked to report either distinctly remembering a previously presented item (a remember response) or merely knowing that the item has been presented (a know response). The proportion of remember responses is reduced in older adults, although the proportion of know responses is either unchanged (Mäntylä, 1993) or is increased (Jacoby, Jennings, \& Hay, 1996). These results suggest that older adults tend to rely on automatic processing and have more difficulty controlling memory processes than do their younger counterparts.

Memory can also be controlled through the judicious use of mnemonic strategies. For example, imagery and verbal 
association strategies have been shown to enhance memory in both the laboratory and real-world situations. Although older adults do not engage in spontaneous strategy use at any greater rate than do younger adults, their use of a strategy can greatly enhance performance if it is suggested to them (see West, 1996, for a review). The use of strategies requires strong motivation and effort, and although older adults can often see the benefits of using such strategies in the short term, the maintenance of strategy use is short lived in the real world if motivation and effort are not rewarded (Dunlosky \& Hertzog, 1998; West, 1996). However, it seems likely that the ability to remember important information is critically dependent on the development and maintenance of an efficient strategy, and for this reason, it is of interest to examine more closely the extent to which younger and older adults can make use of such strategies.

Also of relevance is the work on age-related differences in working memory. The literature clearly indicates that older adults perform less well on virtually all working memory tasks (see Craik \& Jennings, 1992; Moscovitch \& Winocur, 1992; Zacks, Hasher, \& Li, 2000, for reviews). The reasons behind these age-related differences are less clear; they could be a consequence of reduced processing speed (Salthouse, 1994), less efficient inhibitory processes (Hasher \& Zacks, 1988), or reduced processing resources (Craik \& Byrd, 1982), but the decline itself is beyond dispute, and it holds over a wide variety of tasks.

To test the effects of aging on the ability to remember important events, we used a paradigm that brings the value of remembering events under experimental control (Watkins $\&$ Bloom, 1999). Words were randomly assigned values, or arbitrary "points," and the subjects were instructed to maximize the point value of their recall. In the initial experiments reported here, the subjects were given lists of 12 words, with each word randomly paired with a different value, ranging from 1 to 12 . Following recall of each list, the subjects were informed of their score, which was the sum of the point values of the recalled words. The subjects were told that the goal was to keep this score as high as possible and to maximize the overall "value" of their memory. Using a selectivity index (SI) developed by Watkins and Bloom, we measured the degree of selectivity that a subject displayed. This selectivity index is based on the subject's score (the sum of the points that were paired with the recalled items, or the "value" of the recalled items), relative to chance and ideal performance. This equation takes into account the subject's score relative to an ideal score that represents recall of only the most highly valued words at that level of recall:

$$
\text { SI }=\frac{\text { subject's score }- \text { chance } \text { score }}{\text { ideal score }- \text { chance } \text { score }}
$$

For example, if a subject remembered four words, and the points associated with the words were $12,10,9$, and 8 , that subject's SI would be considered quite high. The ideal score if one remembers four words is $12+11+10+9=$ 42 , whereas the score of the subject in question is 39 .
Chance score is based on calculating the average value of the points (using a 12-word list, with numbers ranging from 1 to 12, the average would be 6.5) and multiplying that value by the number of words recalled (in this case, four). Thus, the SI in this case is $(39-26) /(42-26)=$ .81 . If on a second list, the subject recalls 1 word (the 10point word), their SI would be $(10-6.5) /(12-6.5)=$ .64. In Equation 1, the chance score is subtracted from both the numerator and the denominator (as a correction for guessing) in order to assign chance performance a score of 0. Perfect selectivity would result in an SI of 1.0, whereas selection of words with the lowest values (e.g., recalling the 1-, 2-, and 3-point words) would result in an SI of -1.0 . A set of words recalled with no regard to their point values (i.e., showing no selectivity) would result in a selectivity index close to 0 .

The research reviewed above suggests that older adults will not only recall fewer words, but will also be less selective. This hypothesis is based on the notion that, in general, older adults appear to have more difficulty controlling memory processes. If this hypothesis is true, we would expect the older adults to have greater difficulty maintaining a high level of selectivity, relative to the younger adults, regardless of how many words are successfully remembered.

\section{EXPERIMENT 1}

The purpose of Experiment 1 was to assess differences in recall and selectivity for younger and older subjects, using the Watkins and Bloom (1999) technique. It was expected that the older adults would not only recall fewer words, but be less selective in their recall than the younger adults.

\section{Method}

\section{Subjects}

The subjects were 18 undergraduate students from the University of Toronto ( 14 women, 4 men; mean age $=20.3$ years, mean number of years of education $=14.1$, who received course credit for participation, and 18 older adults ( 11 women, 7 men; mean age = 71.7 years, mean number of years of education $=15.3$ ), who were offered $\$ 10$ each for their participation.

\section{Materials and Design}

The stimulus words were 648 nouns. Each contained no more than five letters and had an everyday occurrence of at least 30 times per million according to the Thorndike-Lorge count (Thorndike \& Lorge, 1944). They were randomly assembled into fifty-four 12word lists, of which 6 were used for practice and 48 for the experiment proper. SuperLab Pro, implemented on a PC-compatible computer, was used to run the experiment.

Three versions of 48 lists were created, each with a separate randomization. Within each list, each word was assigned a unique number between 1 and 12. Regardless of the practice lists, the order of the numbers was random with the Latin square constraint that each within-list position assumed each value just once in each successive block of 12 lists.

\section{Procedure}

The subjects were tested individually. They were told that on each trial they would be presented with a series of 12 words and that each 
word would be paired with a number, or point value, between 1 and 12. They were told that their task was to try to remember the words paired with the highest point values, so that they would maximize the sum of the point values of the recalled words. The subjects were also informed that they would be told their score after recall of each list. The experimenter then answered any questions and informed the subjects that they would begin with a practice session. Three practice lists were given, and after this, any remaining questions were answered. The subjects then began the experimental session, which consisted of four blocks of 12 lists. A short break was given between blocks, and each block after the first was preceded by an additional practice list.

Each word and point value was presented for $1 \mathrm{sec}$ in the center of the computer screen, in 32-point Times New Roman typeface, and was followed directly by the next word-value pair. The 12th pair was followed by the word recall shown in capital letters in the center of the screen. The subjects were then given $20 \mathrm{sec}$ to call out as many of the words as they could remember. The experimenter, who sat behind the subject, out of sight, recorded the words and announced the subject's score, which was the sum of the point values paired with the recalled words. The subjects initiated the next trial by pressing the space bar.

\section{Results and Discussion}

The results for memory performance and selectivity are shown in the top half of Table 1. As predicted, the younger adults recalled more words than did the older adults $[t(34)=$ $4.09, p<.001]$. However, contrary to our prediction, the SI was significantly greater for the older adults than for the younger adults $[t(34)=2.10, p<.05]$. This is a surprising result that appears to contradict the evidence that shows that older adults have less cognitive control over their memory processes than do their younger counterparts. Some possible reasons for this discrepancy will be discussed after we describe two further experiments. One possibility is that the older adults used a perceptual/ attentional strategy to limit memory input to only a few highly valued items. This possibility was examined in Experiment 2 , in which point values were not presented until after the offset of each word, thereby negating the possibility of the subjects' deliberately avoiding perceptual and attentional contact with particular words.

\section{EXPERIMENT 2}

One way in which subjects can control which words they will recall is to restrict their attention to the high-value words. Perhaps the older adults in Experiment 1 were

Table 1

Mean Number of Words Recalled and the Mean Selectivity Index for Younger and Older Adults in Experiments 1 and 2

\begin{tabular}{|c|c|c|c|c|}
\hline \multirow[b]{2}{*}{ Age Group } & \multicolumn{2}{|c|}{ Words Recalled } & \multicolumn{2}{|c|}{$\underline{\text { Selectivity Index }}$} \\
\hline & $M$ & $S D$ & $M$ & $S D$ \\
\hline \multicolumn{5}{|c|}{ Experiment 1} \\
\hline Young & 4.79 & 0.88 & .58 & .20 \\
\hline Old & 3.80 & 0.53 & .72 & .23 \\
\hline \multicolumn{5}{|c|}{ Experiment 2} \\
\hline Young & 5.38 & 0.87 & .54 & .12 \\
\hline Old & 3.81 & 0.68 & .68 & .16 \\
\hline
\end{tabular}

more prone to adopt a strategy of simply disregarding all except the high-value words. If so, their selectivity advantage should not hold if the value of each word is withheld until after the word has disappeared from the screen, since under these conditions, the words would have to be attended to and retained at least until their values are revealed. In the present experiment, the value for each word was presented after the word had disappeared from the screen, forcing the subject to read the word and briefly remember it. If the older subjects were directing their attention only to high-value words (and paying little attention to the low-value words) in the previous experiment, and this strategy led to superior selectivity, the age-related effect should disappear or be reversed in Experiment 2.

\section{Method}

\section{Subjects}

The subjects were 18 undergraduate students from the University of Toronto ( 10 women, 8 men; mean age $=20.7$ years, mean number of years of education $=14.3$ ), who received course credit for their participation, and 18 older adults ( 9 women, 9 men; mean age $=68.9$ years, mean number of years of education $=14.2$ ), who were offered $\$ 10$ each for their participation.

\section{Materials and Design}

Four versions of twenty-eight 12-word lists were constructed from the words used in Experiment 1. Four of these lists were used for practice and 24 for the experiment proper. The design was the same as in Experiment 1.

\section{Procedure}

The procedure was similar to that in Experiment 1, with one modification. In this experiment, each word was presented for $1 \mathrm{sec}$ and was immediately followed by its value, which also was presented for $1 \mathrm{sec}$. Thus, instead of the words and numbers appearing together on one screen, they appeared consecutively, ensuring that the subject would attend to and briefly retain each word before the value of the word was revealed. Following the presentation of the 12 words and numbers, the subjects recalled the words in the same manner as that described in Experiment 1.

\section{Results and Discussion}

The results for both age groups are displayed in the bottom half of Table 1. The results followed the same general trend as that seen in Experiment 1. The younger adults recalled more words than did the older adults $[t(34)=6.02$, $p<.001]$. The mean SI for the older adults was significantly greater than the mean SI for the younger adults $[t(34)=3.01, p<.01]$. The present experiment thus replicated the essential finding from Experiment 1, suggesting that even when each word in the list must be processed until a value is presented, older adults are more selective about what information is later remembered. Thus, the older adults' higher SI cannot be attributed to a strategy of totally disregarding the less valuable words.

\section{EXPERIMENT 3}

Given the surprising findings from Experiments 1 and 2, a third experiment was conducted for several reasons. Two control conditions were added. In one, only words were 
presented (words only condition); in the other, both words and numbers were presented, but the subjects were told to ignore the numbers and simply attempt to recall as many words as possible (ignore numbers condition). In both of these new conditions, it was expected that the younger adults would recall more words than would the older adults, but selectivity would be at chance levels for both groups, since in one condition, the number values were not visible, and in the other condition, the number values were not important. It was also of interest to see whether the younger and older adults could increase performance on either selectivity or the number of words recalled simply by being given instructions as to what measure should be emphasized. It was expected that the older adults would have difficulty improving performance when one measure (words or selectivity)was emphasized, whereas the younger adults would be better at shifting their attention to either remembering more words or trying to keep their selectivity as high as possible. Thus, it was of interest to see whether selectivity was a process that could be controlled on the basis of what information is emphasized as being important and what feedback is given regarding performance. Most important in terms of testing reasons for the older adults' superior SI in the previous experiments, it is possible that the older adults were placing a greater emphasis on being selective and that this came at the cost of remembering fewer words. If this was the case, we would expect to see a larger tradeoff in the older adults between overall recall and selectivity.

\section{Method}

\section{Subjects}

The subjects were 16 undergraduates from the University of Toronto ( 10 women, 6 men; mean age $=19.1$ years, mean number of years of education $=13.3$ ), who received course credit, and 16 older adults ( 11 women, 5 men; mean age $=74.3$ years, mean number of years of education $=14.7$ ), who received $\$ 10$ each for their participation.

\footnotetext{
Materials and Design

The materials were the same as in Experiment 1. Four versions of 48 lists were created, with a separate randomization for each version. All other specifications were the same as in Experiment 1. The order of the two control and two emphasis conditions was counterbalanced so that each condition appeared once in each of the four blocks across each group of 4 subjects. The materials used in each condition were also counterbalanced so that each block of words was used equally often in each of the four conditions. The subjects were given one practice list prior to the beginning of each block.
}

\section{Procedure}

The subjects were warned that the instructions would be modified from time to time. For the block of lists assigned to the words only condition, they were shown the words without values and were told to remember as many words as they could; after each list, they were told how many words they had correctly recalled. For the other three conditions, the values were shown along with the words, just as in Experiment 1. For the ignore numbers condition, they were told that the numbers meant nothing. The task and feedback were the same as in the words only condition. In the value and words and value conditions, they were told that the numbers represented the values of the words. In the value condition, they were to maximize the value of their recall (as in Experiments 1 and 2); in the words and value condition, they were to give equal emphasis to the number of words recalled and to the value of their recall. To reinforce these instructions, feedback was restricted to the total value of the recalled words in the value condition and included both the number of words recalled and their total value in the words and value condition.

\section{Results and Discussion}

Table 2 shows the mean number of words recalled and the mean selectivity score for each group of subjects under each of the four conditions. For each condition, the younger adults recalled more words than did the older adults $[t(30) \geq 2.83, p<.01]$ in each case. The control conditions confirmed that when numbers were absent or were to be disregarded, selectivity was near chance for both groups. In terms of selectivity, the older adults showed greater selectivity than did the younger adults when both groups were told to maximize both their scores and the number of words recalled $[t(30)=2.82, p<.01]$. When only score was emphasized, the older adults again showed greater selectivity, although this result was not statistically significant $[t(30)=1.46, p=.16]$.

In order to check for the possibility of a tradeoff between selectivity and number of words recalled, the number of words recalled in the condition in which only words were presented was compared with the number of words recalled in the emphasize score condition for both the younger and the older adults. For the young group, recall dropped from 4.74 words in the words only condition to 4.38 words in the emphasize score condition, a drop of $8 \%$. The corresponding figures for the older adults were 3.94 words and 3.61 words, also a drop of $8 \%$. These results demonstrate that the two groups traded off a similar number of words when score was emphasized. There is thus no evidence to suggest that the older adults traded off more recall quantity in order to be more selective.

Given the consistent findings from the three present experiments, it is clear that the surprising superiority of the older adults in this type of selectivity is not tied to a particular selection of words or experimental subjects. It is a stable and replicable phenomenon. In order to gain greater insight into the age-related differences underlying the results, the data from Experiments 1, 2, and 3 were tabulated as proportions of words recalled by the two age groups as a function of point values. These data are shown in Table 3 .

One striking aspect of performance that is shown in Table 3 is that the subjects from both age groups demon-

Table 2

Mean Number of Words Recalled and the Mean Selectivity Index for Younger and Older Adults in Each of the Four Conditions in Experiment 3

\begin{tabular}{|c|c|c|c|c|c|c|c|c|}
\hline \multirow[b]{3}{*}{ Condition } & \multicolumn{4}{|c|}{ Words Recalled } & \multicolumn{4}{|c|}{ Selectivity Index } \\
\hline & \multicolumn{2}{|c|}{ Young } & \multicolumn{2}{|c|}{ Old } & \multicolumn{2}{|c|}{ Young } & \multicolumn{2}{|c|}{ Old } \\
\hline & $M$ & $S D$ & $M$ & $S D$ & $M$ & $S D$ & $M$ & $S D$ \\
\hline Words only & 4.74 & 0.49 & 3.94 & 0.60 & .01 & .07 & -.01 & .06 \\
\hline Words and numbers & 4.66 & 0.51 & 3.75 & 0.40 & .07 & .14 & .05 & .09 \\
\hline Emphasize score & 4.38 & 0.73 & 3.61 & 0.47 & .66 & .19 & .76 & .21 \\
\hline Emphasize both & 4.32 & 0.61 & 3.76 & 0.51 & .46 & .25 & .68 & .16 \\
\hline
\end{tabular}


Table 3

Mean Proportions of Words Recalled for Each Word Point Value for Younger and Older Adults in Experiments 1, 2, and 3, and Rank-Order Correlations $(\rho)$ Between Point Values and Proportions Recalled

\begin{tabular}{|c|c|c|c|c|c|c|c|c|c|c|c|c|c|}
\hline \multirow[b]{2}{*}{ Group } & \multicolumn{12}{|c|}{ Point Value of Word } & \multirow[b]{2}{*}{$\rho$} \\
\hline & 1 & 2 & 3 & 4 & 5 & 6 & 7 & 8 & 9 & 10 & 11 & 12 & \\
\hline \multicolumn{14}{|c|}{ Experiment 1} \\
\hline Young & .14 & .12 & .16 & .17 & .19 & .27 & .39 & .53 & .63 & .71 & .70 & .70 & +.97 \\
\hline Old & .05 & .05 & .06 & .06 & .07 & .14 & .21 & .40 & .51 & .72 & .71 & .73 & +.99 \\
\hline \multicolumn{14}{|c|}{ Experiment 2} \\
\hline Young & .18 & .15 & .21 & .27 & .28 & .38 & .43 & .61 & .66 & .72 & .77 & .75 & +.99 \\
\hline Old & .06 & .01 & .06 & .06 & .10 & .18 & .24 & .45 & .54 & 69 & .67 & .72 & +.97 \\
\hline \multicolumn{14}{|c|}{ Experiment 3: Words only } \\
\hline Young & .36 & .39 & .34 & .40 & .32 & .35 & .35 & .44 & .41 & .46 & .40 & .40 & +.57 \\
\hline Old & .31 & .33 & .26 & .33 & .21 & .27 & .33 & .31 & .31 & .38 & .29 & .40 & +.33 \\
\hline \multicolumn{14}{|c|}{ Experiment 3: Words and Numbers } \\
\hline Young & .47 & .32 & .35 & .40 & .40 & .42 & .36 & .43 & .44 & .36 & .36 & .44 & +.18 \\
\hline Old & .33 & .32 & .32 & .33 & .39 & .33 & .32 & .33 & .34 & .33 & .29 & .29 & -.19 \\
\hline \multicolumn{14}{|c|}{ Experiment 3: Emphasize Score } \\
\hline Young & .08 & .09 & .09 & .11 & .05 & .21 & .35 & .53 & .62 & .74 & .69 & .79 & +.92 \\
\hline Old & .05 & .03 & .03 & .05 & .05 & .07 & .18 & .32 & .49 & .77 & .79 & .77 & +.94 \\
\hline \multicolumn{14}{|c|}{ Experiment 3: Emphasize Both } \\
\hline Young & .17 & .21 & .16 & .17 & .16 & .26 & .34 & .42 & .49 & .63 & .65 & .65 & +.89 \\
\hline Old & .06 & .08 & .10 & .09 & .11 & .18 & .22 & .34 & .53 & 67 & .64 & .69 & +.99 \\
\hline
\end{tabular}

strated excellent sensitivity to point values in their patterns of recall. This sensitivity is reflected not only in the comparatively high recall of words valued at 10,11 , and 12 , but also in the systematic increase in recall across the whole range of values from 1 to 12 . The reliability of these trends was indexed by computing Spearman rank-order correlation coefficients $(\rho)$ between point values and proportions recalled by each group for the various conditions in the three experiments. The values of $\rho$ shown in Table 3 range between +.89 and +.99 for the young group and between +.92 and +.99 for the old group, for the four conditions in which point values were relevant. It is noteworthy first that this sensitivity extends throughout the range of point values; it was not restricted to the highest valued words that may have been selected and kept in mind throughout presentation. Second, the sensitivity to point values (as indexed by the correlation coefficients) is as high in the older adults as in the younger adults. These findings will be examined further in the General Discussion section. A final feature of the correlation coefficients shown in Table 3 is that the values of $\rho$ are much lower for the two conditions in which point values were not relevant (in Experiment 3, words only and words plus numbers conditions). In those conditions, there was no reason to expect a relation between point value and recall, and three of the four correlations were nonsignificant. The significant value of $\rho$ for the younger subjects (words only), $\rho=$ $+.57(p<.05)$, is anomalous and might have been due to the somewhat easier words' being allocated by chance to higher nominal point values.

For the four conditions in which points were relevant (in Experiments 1, 2, and 3: emphasize score, and Experiment 3: emphasize both), Table 3 shows that recall levels were consistently and equivalently high for words with point values of 10,11 , and 12 , but then dropped progressively from point value 9 to point value 1 . The table also shows that the older adults recalled as many words with values of 10,11 , and 12 as did the younger adults in the same four conditions (mean recall proportions are .71 for both groups). Bear in mind that words with values of 10, 11, and 12 could occur anywhere in the list - that is, they were typically not the last three words in any list. Nonetheless it seems very likely that the subjects maintained highvalue words in mind (or in "primary memory") as the words were presented and then output them first. Unfortunately, we did not record output order in these studies. On the assumption that recall of words with values between 10 and 12 represents retrieval from primary memory in the sense of Waugh and Norman (1965) and that recall of words with values between 1 and 9 represents retrieval from secondary memory, Table 3 thus shows that primary memory recall was equivalent for the two age groups but that secondary memory recall levels were higher for the younger adults. This pattern of equivalent primary memory recall with an age-related decrement in secondary memory recall is in line with previous reports (see, e.g., Craik, 1968; Craik \& Jennings, 1992).

This consistent pattern of age-related differences in recall could also provide an answer to the puzzle of why older adults have higher selectivity scores. That is, if both groups recall 2-3 high-value words from primary memory, but the younger adults augment this initial output with more words from secondary memory than do their older counterparts, the younger adults could be "diluting" their SI by recalling additional words of lower value. As a hypothetical example, let us assume that both groups re- 
called items valued at 10,11 , and 12 , and that the young adults recalled two further words at random from the remainder, but the older adults recalled only one further word at random from the remainder. This plausible scenario would result in a selectivity score of .75 for the older group $(10+11+12+5=38-26 / 42-26)$ but only .60 for the younger group $(10+11+12+6+4=43-32.5 /$ $50-32.5)$.

This scenario could well be too extreme; the orderly decline in proportions of words recalled from point value 9 to point value 1, as is shown in Table 3, makes it likely that the subjects recalled higher valued words from those words that remained. Nevertheless, any deviation from "ideal recall" (i.e., always recalling the highest valued word from those remaining) will reduce the SI from the high value achieved by recalling a few words from the top 3 or 4 . Since the subjects were instructed to maximize their score-defined as the total number of points assigned to recalled words-it would be a rational strategy for them to recall as many words as possible, even those with point values of 1,2 , or 3 . If this strategy was pursued more effectively by the younger adults in the present experiments, it would have increased the number of words recalled, but reduced the SI, relative to the older group. This general account of the results was tested in a fourth experiment in which primary memory recall was largely eliminated by interpolating an arithmetic task between word presentation and recall. Under these conditions, recall reflects retrieval from secondary memory only, and the somewhat artificial advantage of the older subjects' selectivity scores should be eliminated.

\section{EXPERIMENT 4}

The purpose of Experiment 4 was to examine the degree to which younger and older adults are able to be selective in a task that requires the use of long-term or secondary memory. In the present experiment, the subjects were given a longer list than in the previous experiments ( 20 words instead of 12), and each word was presented for a longer duration $(4 \mathrm{sec}$ instead of $1 \mathrm{sec})$. Furthermore, in order to examine secondary memory specifically, two other conditions were added: an immediate free recall test and a delayed recall test. The subjects recalled the words either immediately after presentation (no-delay condition) or after a 1-min arithmetic task (delay condition). The reason for incorporating a delay condition was to reduce the contribution of primary memory and force both the younger and older adults to recall words largely from secondary memory.

\section{Method}

\section{Subjects}

The subjects were 24 undergraduates from the University of Toronto ( 14 women, 10 men; mean age $=21.9$ years, mean number of years of education $=15.5$ ), who were either paid $\$ 10$ or received course credit, and 24 older adults ( 17 women, 7 men; mean age $=$ 69.0 years, mean number of years of education $=14.8$ ), who received $\$ 10$ each for their participation.

\section{Materials and Design}

Four lists were created by using a random assortment of words from the previous experiments. Each list contained 20 words paired with numbers ranging from 1 to 20 . The serial position of each word-number pair was randomized across the four lists, and two different versions of each of the four lists were constructed with different serial orders and word-number pairs. Each subject received four lists, and the order of the delay and no-delay conditions was counterbalanced, so that half of the subjects began with a list in the no-delay condition $(\mathrm{ABAB})$, and the other half began with a list in the delay condition (BABA). In total, each subject received two lists in the no-delay condition and two lists in the delay condition, and the order of each list was also counterbalanced across subjects.

\section{Procedure}

The subjects were tested individually. They were told that on each trial they would be presented with a series of 20 words and that each word would be paired with a number, or point value, between 1 and 20. They were told that their task was to try to remember the words paired with the highest point values and that they should maximize the sum of the point values of the recalled words. The subjects were also informed that they would be told their score after recall of each list and that their task was to maximize their score. The subjects were told that they would study four lists (ABAB), and after two of the lists (B), they would perform an arithmetic task for $1 \mathrm{~min}$ before performing the recall task. Prior to the presentation of each list, the subjects were told whether they would recall the words immediately following the last word-number pair (no-delay condition) or whether there would be an arithmetic task before recall (delay condition). The experimenter answered any questions, and the subjects then began the experimental session.

Each word and point value was presented for $4 \mathrm{sec}$ in the center of the computer screen, in 32-point Times New Roman typeface, and was followed directly by the next word-value pair. The last pair was followed by a blank screen, and either the subjects were instructed to recall the words (no-delay condition) or they performed an arithmetic task for $1 \mathrm{~min}$ (delay condition). The arithmetic task involved the addition of the number 3 to a spoken list of random numbers that ranged from 1 to 9 . The subjects responded with their answers, and the next number was read to them approximately every $2 \mathrm{sec}$. Following this, the subjects were given 1 min to verbally recall as many of the words as they could. The experimenter, who sat behind the subject and was out of sight, recorded the words and announced the subject's score, which was the sum of the point values paired with the recalled words. The subjects initiated the next list by pressing the space bar.

\section{Results and Discussion}

Table 4 shows the mean number of words recalled and the mean selectivity score for the younger and older subjects in the delay and no-delay conditions. In terms of memory performance, in the no-delay condition, the younger adults recalled more words than did the older adults $[t(46)=5.94, p<.001]$, and in the delay condition, both groups displayed poorer memory performance, with the younger adults again recalling more words than the older adults $[t(46)=5.97, p<.001]$.

In terms of selectivity, the older adults showed greater selectivity than did the younger adults in the no-delay condition $[t(46)=1.93, p<.05$; one-tailed t test $]$, generally replicating the findings from Experiments 1-3. However, in the delay condition, the selectivity scores for the younger and older adults were not statistically different $[t(46)<1$, 
Table 4

Mean Number of Words Recalled and the Mean Selectivity Index for Younger and Older Adults in the No-Delay and Delay Conditions in Experiment 4

\begin{tabular}{|c|c|c|c|c|c|c|c|c|}
\hline \multirow[b]{3}{*}{ Condition } & \multicolumn{4}{|c|}{ Words Recalled } & \multicolumn{4}{|c|}{ Selectivity Index } \\
\hline & \multicolumn{2}{|c|}{ Young } & \multicolumn{2}{|c|}{ Old } & \multicolumn{2}{|c|}{ Young } & \multicolumn{2}{|c|}{ Old } \\
\hline & $M$ & $S E$ & $M$ & $S E$ & $M$ & $S E$ & $M$ & $S E$ \\
\hline No delay & 11.48 & 0.68 & 6.80 & 0.40 & .27 & .06 & .41 & .04 \\
\hline Delay & 10.25 & 0.67 & 5.39 & 0.46 & .38 & .04 & .32 & .05 \\
\hline
\end{tabular}

$p>.05]$ from each other. This suggests that when the younger and older adults were forced to rely only on secondary memory, they displayed no age-related differences in terms of the SI.

In order to assess the degree to which recall was influenced by point values, see the mean proportions recalled by the two groups in Table 5. Since only two lists were presented in each condition, the table shows recall proportions combined over adjacent point values (i.e., $1+2$, $3+4 \ldots 19+20)$. In general, recall declined with declining point values for both groups and in both conditions. Rank-order correlation coefficients $(\rho)$ were calculated, and their values ( $n=10$ in each case) are +.84 , $+.92,+.91$, and +.89 for conditions young-no delay, young-delay, old-no delay, and old-delay, respectively. Thus, there were no consistent differences in selectivity (as measured by the correlations), either between age groups or between the delay and no-delay conditions. All four conditions yielded significant values of $\rho(p<.01)$, however, showing that the subjects were able to discriminate and select higher valued words in all cases.

The main finding from Experiment 4 was that the older subjects obtained a higher SI than did their youngercounterparts under conditions of immediate recall (no delay), thereby replicating the results of Experiments 1, 2, and 3, but the two age groups' SI scores did not differ under conditions of delayed recall. This pattern of results is in line with the suggestion that when primary memory can be used (no delay in this experiment), older subjects maintain 2-3 high-value words in this fashion and augment their primary memory recall with relatively few further (lower valued) words retrieved from secondary memory. Younger subjects, on the other hand, also retrieve 2-3 primary memory words and a relatively larger number of words from secondary memory. These secondary memory words are of relatively low value, however, and this pattern of recall results in a lower SI. Under delayed recall conditions, pri- mary memory is largely eliminated and recall is therefore mainly from secondary memory. In this condition, the young subjects still recalled more words than did the older subjects, but the two age groups showed equivalent selectivity, judged both in terms of the SI and in terms of the correlation between point values and proportions recalled. However, one puzzling finding is that selectivity and the $\rho$ correlations appear to be at least as great after a delay as without a delay, suggesting that both age groups are able to be just as selective when relying solely on secondary memory. This finding might indicate that one can be somewhat selective when relying only on secondary memory, but that when both primary and secondary memory can be used to recall words, selectivity can be impaired, although future experiments need to address this point in greater detail.

\section{GENERAL DISCUSSION}

The purpose of the preceding experiments was to address the question of selectivity in a short-term recall situation and to see whether there are age-related differences in this type of selectivity. Watkins and Bloom (1999) proposed a selectivity index that compares a subject's ability to select and recall high-value words with ideal selection at a given level of recall. Experiments 1 and 2 showed, surprisingly, that older adults achieved significantly higher levels of selectivity, despite their recalling fewer words than did their younger counterparts. This finding was confirmed in Experiment 3 by using variations of the procedure. The notion that the higher levels of selectivity in older adults reflects their greater reliance on recall from primary memory was tested in Experiment 4; that is, older adults might retain two or three high valued words in primary memory, recall those first, and then augment this recall by retrieving only one or two further lower valued words from secondary memory. The younger adults, in contrast, might also recall two or three high-value words from primary memory but augment this recall by retrieving a greater number of words (relative to the older subjects) from secondary memory, thereby "diluting" the quality of their recall and reducing their SI. The results of Experiment 4 support this account by showing that the older subjects again achieved higher selectivity scores than did the younger adults in immediate recall, but that this superiority disappeared when primary memory was substantially eliminated by means of an arithmetic task interpolated between presentation and recall.

Table 5

Proportions of Words Recalled as a Function of Point Value Under Delay and No-Delay Conditions for Younger and Older Adults in Experiment 4

\begin{tabular}{lcccccccccc}
\hline & \multicolumn{10}{c}{ Point Value } \\
\cline { 2 - 11 } \multicolumn{1}{c}{ Condition } & $1-2$ & $3-4$ & $5-6$ & $7-8$ & $9-10$ & $11-12$ & $13-14$ & $15-16$ & $17-18$ & $19-20$ \\
\hline Young: No delay & .45 & .46 & .43 & .41 & .49 & .68 & .65 & .57 & .73 & .80 \\
Young: Delay & .36 & .28 & .24 & .38 & .44 & .59 & .60 & .60 & .77 & .71 \\
Old: No delay & .14 & .14 & .09 & .23 & .27 & .52 & .49 & .32 & .53 & .65 \\
Old: Delay & .10 & .21 & .09 & .17 & .23 & .31 & .30 & .28 & .39 & .44 \\
\hline
\end{tabular}


The previous literature on age-related differences in cognitive control suggests strongly that older adults are less able to control their processing operations. The present results show equivalent control abilities between older and younger adults, and thus provide an interesting exception to the general rule. This equivalence is shown both by the comparable values of the SI, once primary memory recall was reduced or eliminated (Experiment 4), and by the comparable values of the correlations between recall probabilities and point values in all four of the present experiments (see Tables 3 and 5). The data shown in Tables 3 and 5 are striking in that recall probabilities decline progressively throughout the range of point values. It is important to note that the words and their point values were presented for only $1 \mathrm{sec}$ each in Experiments 1 and 3 and for $2 \mathrm{sec}$ each in Experiment 2. This procedure leaves little time for strategic manipulation, yet the older adults were as able as their younger counterparts to utilize the point value information effectively.

The question arises as to whether selectivity reflects encoding or retrieval processes. The present data do not permit a clear answer to this question, but it seems likely that the two major factors relate more to encoding than to retrieval. First, as suggested earlier, subjects might hold highly valued items in mind (in primary memory) during presentation and recall those first. Second, subjects might devote more attention and rehearsal to higher valued words, thereby encoding them more effectively. In summary, the present article reports a novel paradigm with which to explore strategic control processes in memory and provides surprising evidence that older adults use these control processes as effectively as their younger counterparts.

\section{REFERENCES}

CraIK, F. I. M. (1968). Two components in free recall. Journal of Verbal Learning \& Verbal Behavior, 7, 996-1004.

CrAIK, F. I. M., \& BYRD, M. (1982). Aging and cognitive deficits: The role of attentional resources. In F. I. M. Craik \& S. Trehub (Eds.), Aging and cognitive processes (pp. 191-211). Hillsdale, NJ: Erlbaum.

CrAIK, F. I. M., \& JENNINGS, J. (1992). Human memory. In F. I. M. Craik \& T. A. Salthouse (Eds.), The handbook of aging and cognition (pp. 51110). Hillsdale, NJ: Erlbaum.
Dunlosky, J., \& Hertzog, C. (1998). Training program to improve learning in later adulthood: Helping older adults educate themselves. In D. J. Hacker, J. Dunlosky, \& A. C. Graesser (Eds.), Metacognition in educational theory and practice (pp. 249-275). Mahwah, NJ: Erlbaum.

HASHER, L., \& ZACKS, R. T. (1988). Working memory, comprehension and aging: A review and a new view. In G. H. Bower (Ed.), The psychology of learning and motivation (Vol. 22, pp. 193-225). New York: Academic Press.

JACOBY, L. L., \& HAY, J. F. (1998). Age-related deficits in memory: Theory and application. In M. A. Conway, S. E. Gathercole, \& C. Cornoldi (Eds.), Theories of memory II (pp. 111-134). Hove, U.K.: Psychology Press.

JACoby, L. L., Jennings, J. M., \& Hay, J. F. (1996). Dissociating automatic and consciously controlled processes: Implications for diagnosis and rehabilitation of memory deficits. In D. Hermann, C. McEvoy, C. Hertzog, P. Hertel, \& M. K. Johnson(Eds.), Basic and appliedmemory research: Theory in context (Vol. 1, pp. 161-193). Mahwah, NJ: Erlbaum.

Kester, J. D., Benjamin, A. S., Castel, A. D., \& Craik, F. I. M. (2002). Memory in the elderly. In A. D. Baddeley, B. A. Wilson, \& M. Kopelman (Eds.), Handbook of memory disorders (2nd ed., pp. 543-568). London: Wiley.

MäNTY LÄ, T. (1993). Knowing but not remembering: Adult age differences in recollective experience. Memory \& Cognition, 21, 379-388.

Moscovitch, M., \& Winocur, G. (1992). The neuropsychologyof memory and aging. In F. I. M. Craik \& T. A. Salthouse (Eds.), The handbook of aging and cognition (pp. 315-372). Hillsdale, NJ: Erlbaum.

SAlthouse, T. A. (1994). The aging of working memory. Neuropsychology, 8, 535-543.

Thorndike, E. L., \& LoRge, I. (1944). The teacher's word book of 30,000 words. New York: Columbia University, Teachers College Press.

Watkins, M. J., \& BLoom, L. C. (1999). Selectivity in memory: An exploration of willful control over the remembering process. Unpublished manuscript.

WaUgh, N. C., \& Norman, D. A. (1965). Primary memory. Psychological Review, 72, 89-104.

WEST, R. L. (1996). Compensatory strategies for age-associated memory impairment. In A. D. Baddeley, B. A. Wilson, \& F. N. Watts (Eds.), Handbook of memory disorders (pp. 481-500). London: Wiley.

ZACKs, R. T., HASher, L., \& Li, K. Z H. (2000). Human memory. In F. I. M. Craik \& T. A. Salthouse (Eds.), The handbook of aging and cognition (pp. 293-357). Mahwah, NJ: Erlbaum.

Zacks, R. T., RAdVANSKy, G., \& HASHer, L. (1996). Studies of directed forgetting in older adults. Journal of Experimental Psychology: Learning, Memory, \& Cognition, 22, 143-156.

(Manuscript received November 7, 2001; revision accepted for publication June 25,2002 .) 